\title{
La reciente inserción internacional de la industria mexicana y su impacto regional
}

\author{
Arturo Ranfla González \\ Ana María Avilés Muñoz \\ Instituto de Investigaciones Sociales \\ Universidad Autónoma de Baja California
}

\section{Resumen}

El presente trabajo apunta a la transnacionalización de la economía mundial como fuente de procesos nuevos que alteran las formas convencionales de operación de las economías nacionales. Los puntos de vista aquí sostenidos destacan que el país pasa por un proceso de "fronterización", es decir, un cambio en el eje de gravitación de la industria mexicana a partir de 1982. La generalización de esta tendencia obliga a identificar el enraizamiento de estas inversiones que han mostrado un reciente y notable incremento. La generalización del proceso de producción lleva a destacar diferencias regionales, patentizando la complejidad actual que adquiere el diseño de políticas en la mayoría de los países.

Palabras clave: transnacionalización, economía mundial, proceso de fronterización, industria mexicana, empleo.

\section{Astract}

This job points toward the nationalization of world economy as source of new processes that deform the conventional operating forms of national economies. The points of view herein sustained underline that the country is going through a "borderization". It means, a change on the gravitational axis of Mexican industry starting on 1982. Generalizing this trend helps to identify how investments that have shown a recent and remarkable increase are rooted. At mean time, generalizing the production process helps to discover the regional differences and makes patent the current complexity of the politics design at most countries.

Keywords: trans-nationalization, World economics, borderization process, Mexican industry, employment. 


\title{
LA RECIENTE INSERCION INTERNACIONAL DE LA INDUSTRIA MEXICANA Y SU IMPACTO REGIONAL
}

\author{
Por \\ Arturo Ranfla González \\ Ana María Avilés Muñoz*
}

\section{INTRODUCCION}

La revolución tecnológica y la creciente transnacionalización de los procesos económicos en el siglo XX, son la fuente de una serie de procesos nuevos y transformadores en las formas convencionales de operacion de las economías nacionales. Cambios que se ubican en dos grandes planos: el primero, de naturalezatérica, se caracteriza por someter a la teoría ortodoxa de la cconomía internacional a un reexamen de sus principales postulados, cuyas limitaciones para explicar la operación -real- de los mecanismos de producción y operación monetaria y financiera (Germiadis y Michalet, 1984: 20), responden a la consideración excesiva que ésta hace de los países como verdaderos bloques de factores; cl scgundo cambio, que no está cxento de una serie de connotaciones teóricas e ideológicas, se ubica en la práctica de la política económica seguida en la mayoría de los países capitalistas desde mediados de la década de los setentas, tanto desarrollados como en desarrollo, que se traduce en la aplicación de acciones restrictivas en los campos presupuestales y financieros, que apuntan hacia un retorno generalizado del liberalismo. ${ }^{1}$

Los cambios señalados tienen una incidencia en los patrones de organización económica nacional, que son sometidos a una flexibilización y al deterioro de las condiciones generales de la produccion y del consumo al interior de cada uno de los países, y tienen

- Director e investigadora del Instituto de Investigaciones Sociales de la Universidad Autónoma de Baja California.

${ }^{1}$ El retorno al liberalismo que se desarrolla desde la segunda mitad de la década de los setenta, plantea de una manera general: el saneamiento de las finanzas públicas y una austeridad en el manejo de las variables monetarias y financieras en cada uno de los paises, como recurso para recobrar el equilibrio. En términos de su aplicación, estas políticas se convierten en influyentes en los países desarrollados que se van integrando a esta práctica, mientras que en una buena parte de los países en desarrollo, su aplicación deriva de las sugerencias del Fondo Monetario Internacional. 
como efecto combinado, por una parte, un recrudecimiento de la competencia internacional en términos de productividad y empleo, y por la otra, una tendencia generalizada hacia la homogeneización del espacio mundial en términos de producción y mercado.

Regionalmente, este doble proceso sugiere, a largo plazo, un desvanecimiento paulatino de cada uno de los espacios geoeconómicos diferenciados al interior de las paísesparticipantes en el proceso, que en un momento dado, podría encubrir procesos alternos de naturaleza estructural de corto y mediano plazo que se relacionan con el comportamiento de sectores (ramas en una esfera inferior) y con cada uno de los espacios constituidos en regiones (Steindl, 1986: 15). Esto significa que, encuadradas en una tendencia general a la homogeneización sectorial, las regiones no dejan de manifestar sus diferencias de localización y articulación sectorial, que actualmente nos permiten identificar cuando menos dos grandes subconjuntos: el primero de ellos, constituido por regiones "decadentes", en donde se localizan una serie de industrias tradicionales que con frecuencia se identifican orgánicamente con cada una de las regiones (Hudson and Sadler, 1986:172-193; Carney, et al., 1980); y el segundo, por regiones de emergencia reciente ode "procesamiento para la exportación", cuyos objetivos y cualidades se ubican dentro de la lógica de la integración a los procesos de transnacionalización de la producción catalizada por los progresos en la división técnica y geográfica de la producción (Basile y Germiadis, 1984).

En México, tal dualidad puede ejemplificarse en la organización regional de su industria, cuyos espacios de producción y consumo planteaban, hasta hace poco (1982), dos grandes unidades geoeconómicas relativamente autónomas: 1) el mercado y la producción nacional circunscritageográficamente ala mayor parte del país y sustentada en una política de sustitución de importacioncs que configuró la estructura territorial de esta actividad; y 2) la producción para la exportación y el consumo de importaciones, circunscritos casi exclusivamente a la frontera entre México y Estados Unidos, en donde la industrialización se organizó fundamentalmente alrededor de la industria maquiladora (Sándezy Avilés, 1988).

El análisis de esta dualidad de funcionamiento en cl espacio nacional y la tendencia hacia la homogeneización sectorial, que apuntaría de alguna manera a una fronterización del país por la modalidad en que ha insertado este sector, constituye elobjeto central de nuestro documento. Es evidente que este propósito requicre un 
examen más exhaustivo del que podemos pretender realizar aquí, por este motivo, nuestra exposición tiene como objetivo únicamente: plantear un análisis preliminar sobre el origen, los mecanismos y la posibilidad de evaluar el impacto regional que han tenido los cambios en la producción de la industrial mundial en relación con su articulación reciente a la economía mexicana.

\section{ORIGEN}

El análisis de las condiciones actuales de la inserción de México en la economía mundial, plantea la necesidad de establecer la diferencia entre las formas precedentes en la que ésta se había venido dando y las modalidades más recientes. En el pasado, la exportación de materias primas era la forma en que se articulaba el país con los países centrales, relación que se ha identificado con el colonialismo y neocolonialismo ${ }^{2}$, por el que transitó el país desde el siglo XVI hasta mediados del siglo XX; posteriormente, sustentado en un proyecto nacionalista que buscaba un desarrollo económico relativamente autónomo, el país se integró a las economías centrales en el marco de una dependencia tecnológica. Esta relación se dio en varias formas, como fueron: licencias y suministros del conocimiento práctico (know how), importación directa de bienes de capital y de la inversión extranjera directa. En conjunto, estos mecanismos crearon una dependencia de la importación de bienes de capital e intermedios, mismos que se hicieron cada vez más indispensables para asegurar la expansión de la producción nacional.

Se puede decir que el país, como nación, no ha dejado de transitar por un prolongado proceso de articulaciones con la economía mundial en el cual, de exportador de recursos y materias primas, se convirtió en

2 En la historia de México es particularmente significativa la ruptura que se establece entre el colonialismo político y económico, con el cual se identifican el control español y la penetración económica norteamericana. Fue en la segunda mitad del siglo XIX cuando los cambios tecnológicos en el transporte transformaron el espacio geoeconómico existente y lo integraron en nuevos bloques. La instalación del ferrocarril en México es un ejemplo de tales cambios, toda vez que su desarrollo articuló las primeras zonas industriales del medio-oeste y noroeste de los Estados Unidoscon la capital del país; asimismo, modificó las estructuras del comereio exterior que hasta ese momento mostraban un claro dominio de las potencias europeas. Ver por ejemplo: Coatsworth, J. H. El impacto económico de los ferrocarriles en el porfiriato; Taylor, G.R. The transportation revolution; Kapp, B. Les relations exterieures du Mexique (1821-1921) d' aprés les sources francaises. 
poco menos de medio siglo en importador de bienes intermedios y de capital, acentuando su dependencia "... tanto de la estructura productiva de los países avanzados como de los patrones de comercio internacional y de la tecnología disponible" (Trejo, 1987); transformándose el perfil sectorial y espacial del país.

Las transformaciones sectoriales y espaciales recientes.

A nivel de sectores, en los últimos cuarenta años el país se transformó y pasó de actividades predominantemente primarias y de servicios, a la emergencia de un importante sector industrial, como se destaca en las cifras del cuadro siguiente:

CUADRO 1. México. Empleo por sector (1940-1980)

Año Agrop. Extr. Transf. Constr. Elec. Comerc. Servs.

\begin{tabular}{rrrrrrrr}
\hline 1940 & 65.4 & 1.6 & 10.3 & 1.9 & .1 & 7.0 & 13.4 \\
1950 & 58.3 & 1.2 & 12.8 & 3.4 & .3 & 8.5 & 15.2 \\
1960 & 54.0 & 1.2 & 13.9 & 3.6 & .3 & 9.5 & 16.9 \\
1970 & 41.1 & 1.5 & 18.0 & 4.5 & .4 & 9.7 & 24.3 \\
1980 & 37.0 & 3.0 & 16.7 & 8.4 & .7 & 11.2 & 22.7 \\
\hline
\end{tabular}

FUENTE: VI, VII,I VIII, IX y X Censos Generales de Población y Vivienda. INEGI.

A los cambios graduales en la distribución del empleo se agregan cambios en la distribución del producto interno por sector, en ésta los servicios participaron en promedio con el $25.2 \%$ durante el período 1970-1985; el comercio en la misma proporción y las manufacturas con el $24.2 \%$. Esta reestructuración sectorial se produjo a partir de una reducción sistemática en el producto de las actividades agropecuarias (INEGI, 1986).

Esta transformación sectorial en el país trajo consigo cambios en su organización espacial, al producirse una urbanización cuantitativa y cualitativa muy rápida, que provocó una reconcentración de la población que transformó el patrón de la distribución de la misma en cuarenta años (figura 1). Puede decirse que el perfil sectorial y espacial de país pasó a ser, en poco menos de medio siglo, un proceso 
que muestra una marcada correlación entre la distribución sectorial y la población, tal como lo confirma el cuadro siguiente.

CUADRO 2. México: Coeficientes de determinación de la especialización y concentración (1940-1980)

Año Agric. Extrac. Transp. Constr. Elec. Comerc. Servs.

\begin{tabular}{|c|c|c|c|c|c|c|c|}
\hline 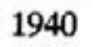 & 0.7444 & 0.02 & 0.7131 & 0.6 & 0.5901 & 0.8346 & 0.7167 \\
\hline 95 & 0.77 & 0.002 & & & & & \\
\hline 96 & 0.80 & 0.00 & 0.0 & 0.7 & 0.1025 & 8 & 0.7992 \\
\hline 97 & 0.88 & 0.00 & $A C$ & 2 & 0.3 & 0.8 & 84 \\
\hline 198 & 0.8201 & 0.0853 & $0^{\prime} 4613$ & 0.5969 & 0.3040 & 0.4131 & 0.5623 \\
\hline
\end{tabular}

FUENTE: Coeficientes de determinación (R2), calculados en base a coeficientes de concentración (Qc) y cocientes de especialización (Oe) por entidad federativa. En base al empleo por sector y la distribución de población que arrojan lo censos de pobalción y vivienda VI, VII, VIII, IX y X. INEGI-SPP.

Relevo o reorientación de la estrategia de desarrollo industrial.

Es posible señalar que el intento por lograr la conformación y consolidación de una economía nacional relativamente autónoma, que se buscaba a través de la política de sustitución de importaciones, se tradujo en un doble flujo: el tradicional, de exportación de recursos naturales y productos agrícolas que generaban divisas; y otro en crecimiento, de bienes de capital e intermedios que eran introducidos para consolidar una planta industrial nacional ${ }^{3}$.

Paralelamente a este doble flujo entre bienes primarios e industriales que configuraron la relación determinante en el modelo de desarrollo nacional (1940-1982), se puede indentificar el origen de la forma reciente de inserción de México en la economía mundial, que a diferencia de las precedentes basadas en el intercambio, se va a integrar directamente a lainternacionalización de la producción. Esta

3 "... se asistía a un agotamiento gradual de las posibilidades más 'fáciles' de sustitución de importaciones y se comenzaba a enfrentar obstáculos de mercado y de tecnología para continuar el proceso de sustitución. Por otro lado, surgían factores de inflexibilidad en la balanza de pagos debido a que las compras en el exterior eran bienes de capital y productos intermedios indispensables para el crecimiento." (Guillén Romo, 1988:91). 


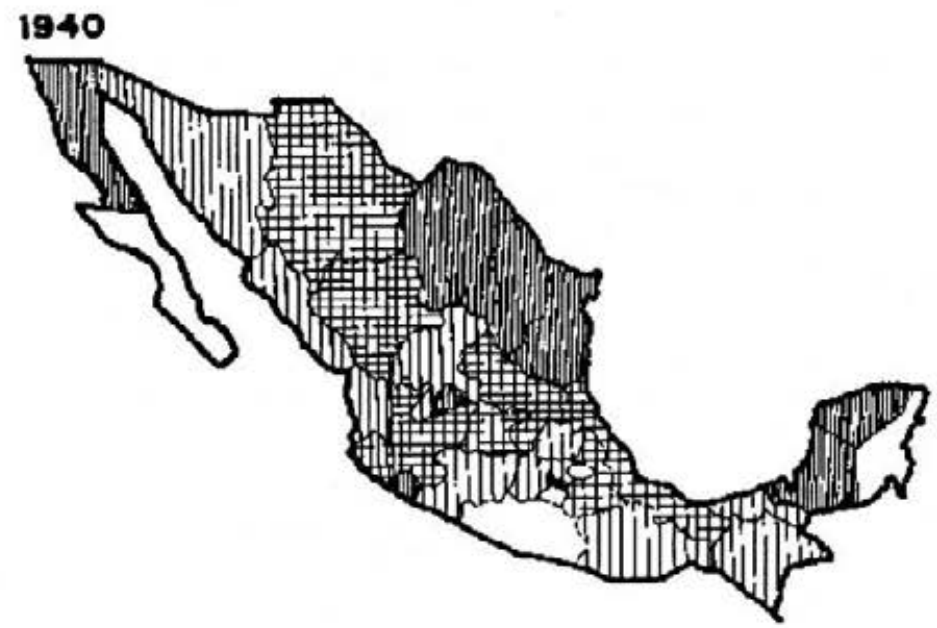

1980

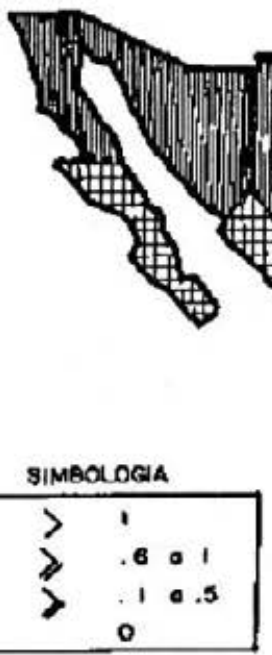

FIGURA 1. México: Cambios en la concentración territorial de la población. (1940-1980). 
inserción que se identifica con el programa de industrialización fronterizo, puesto en marcha en 1965 , se justifica internamente por las condiciones de desempleo regional, que se consideraba que se iban a presentar en la zona fronteriza del norte del país, cuando llegara a su fin el programa de trabajadores agrícolas huésped.

Externamente, el programa se fundamenta en el despliegue, que desde principios de la década de los setenta, realizó la industria de los países centrales hacia algunos países en desarrollo, en los que se aprovecharon las condiciones favorables de inversion, las ventajas de emplazamiento y la existencia de una fuerza de trabajo inagotable, productiva, barata y obediente (Frobel, et al.; 1981: 449), que propició la elevación de los niveles de productividad de las empresas y la capacidad de competir en el mercado mundial.

En suma, pueden destacarse las diferentes formas de articulación que México ha tenido a lo largo de su historia con la economía mundial y la complejidad que ésta ha venido adquiriendo con el ticmpo; sin embargo, es posible distinguir dos grandes momentos: el primero, que corresponde a la internacionalización del comercio en respuesta a una realidad constituida por espacios geográfica y económicamente aislados; el segundo, que pone en evidencia la temprana e importante inscrciónque ha experimentado la cconomía mexicana en la internacionalización de la producción, particularmente de la industria, y que obedece a una realidad, cuyos trazos significativos son la existencia de grandes firmas oligopólicas o monopolistas, la exportación de capitales en una diversidad de modalidades de inversión, la transferencia de tecnología, las diferencias salariales, los cambios en la división internacional del trabajo prevaleciente provocados por la relocalización de los procesos productivos que ésta experimenta, no sólo en sectores modernos, sino también en tradicionales.

\section{MECANISMOS.}

La internacionalización de la producción, que también se ha denominado "la nueva división internacional del trabajo", es un proceso cuyos orígenes económicos son catalizados por el cambio tecnológico que ocurre en un medio ambiente económico mundial, cuya extremada volatilidad obliga a que las firmas que operan a esta escala, desarrollen sumultáneamente medidas de defensa contra los estados donde se localizan sus filiales, así como iniciativas estratégicas 
con el objeto de protegerse de la concurrencia internacional ${ }^{4}$. Esta actitud está estrechamente ligada, y de cierta manera condicionada, por las decisiones y posiciones que asuman cada uno de los estados, entre los cuales estas firmas articulan sus estrategias.

La estrecha vinculación que mantienen las decisiones de inversión con respecto a los agentes estado-nación y las firmas, plantea la necesidad de analizar y evaluar los mecanismos en que se ha desarrollado el proceso de inserción reciente de México en la producción mundial en relación a ambos agentes. Esto sugiere que, analíticamente, se establezca el ámbito propio de las condiciones macroeconómicas y microeconómicas, para aclarar y evaluar la naturaleza de las estrategias de desarrollo industrial que presenta actualmente el país.

\section{Las variables macroeconómicas.}

La inestabilidad de las tasas de cambio internacional desde principios de la década de los setenta, las crecientes fluctuaciones de las tasas de interés en los mercados internacionales yla globalización de la competencia oligopólica entre firmas, se encuentran entre los principales factores macrocconómicos que facilitaron la opcración y desarrollo de la internacionalización de la producción mundial. En México, se estimularon las actividades maquiladoras con el inicio de la flexibilización de las variables monetarias y financieras, que se inició a partir de la devaluación de agosto de 1976; sin embargo, este crecimiento fue limitado por las tendencias recesivas que aparecieron abiertamente en la mayoría de los países desarrollados desde 1973 (OECD, 1988a).

La industria maquiladora creció erráticamente bajo condiciones externas desfavorables, debido a la contracción en la mayor parte de las economías centrales, y en condiciones internas de expasión en los niveles de ingreso y consumo, provocada por los numerosos empréstitos que la banca internacinal le otorgó a México a finales de

${ }^{4} \mathrm{~L}$ a diferencia de ambos mecanismos responde a las definiciones manejadas por Oman (1984), en esencia, la actitud defensiva de las empresas internacionales se refleja en la actitud de importantes empresas líderes por retener o ganar mereados extranjeros, frente al incremento de barreras comerciales impuestas por los estados. Por su parte, las iniciativas estratégicas derivan primordialmente de condiciones nacionales específicas, que se reflejan en condiciones salariales, de ingreso, fiscales y otras, que incrementan las condiciones favorables de las inversiones, tanto de nacionales como de extranjeros. 
la década de los setenta y principios de los ochenta, esto le permitio mantener subsidiados la industria y el tipo de cambio. En estas condiciones, la industria maquiladora experimentó un verdadero auge al inicio de la década de los ochenta, cuando en Estados Unidos, principal mercado, se reactivó el crecimiento del producto interno, se redujo la inflación y se elevaron las tasas de interés, con lo cual este pais captó mayores volúmenes de ahorro internacional, que lo consolidaron como la locomotora de la economía mundial por la magnitud de su consumo (OECD, 1988b: 67) .

Cuadro 3. México: Principales destinos geográficos de las exportaciones totales.

-porcentajes-

\begin{tabular}{lrrr}
\hline DESTINO & 1978 & 1980 & 1984 \\
\hline Países desarrollados & $\mathbf{8 1 . 7}$ & $\mathbf{8 5 . 4}$ & $\mathbf{8 5 . 7}$ \\
$\quad$ Estados Unidos & 68.1 & 64.7 & 58.4 \\
Otros & 13.6 & 20.7 & 27.3 \\
Países en desarrollo & $\mathbf{1 5 . 1}$ & $\mathbf{1 2 . 6}$ & $\mathbf{1 0 . 3}$ \\
$\quad$ América Latina & 9.7 & 6.9 & 6.2 \\
Otros & 5.4 & 5.7 & 4.1 \\
URSS, Europa del Este,Cuba & $\mathbf{0 . 6}$ & $\mathbf{0 . 4}$ & $\mathbf{0 . 6}$ \\
No especificados & $\mathbf{2 . 6}$ & $\mathbf{1 . 6}$ & $\mathbf{3 . 4}$ \\
\hline
\end{tabular}

FUENIE: FMI. Trade Statistics, Yearbook, 1985 y 1986.

Junto a cstas condiciones externas favorablesy a la crisis de la deuda de agosto de 1982, surgen internamente cambios sustantivos en las políticas económica, comercial, financiera y monetaria; sus prácticas se orientaron a fomentar una mayor apertura de México hacia el exterior.

En lo particular, la política cambiaria tuvo un papel determinante en la estrategia de reordenamiento económico implementada por la administración del presidente Miguel de la Madrid; desde el inicio de su gobierno, se consideró que esta variable era determinante para

5 "... A particularly powerful magnet drawing imports to the United States, by far de newly industrialising contries (NICs') biggest market, was the high dollar rate of the early 1980's." 
lograr mejores resultados con respecto al exterior, manteniendo un “...tipo de cambio realista y una existencia suficiente de divisas en el país para permtir el desarrollo de las transacciones comerciales y financieras de la economía con el exterior" (Presidencia de la República, 1985: 31).

Se reconoce que el manejo de esta política cambiaria fue clave para el desarrollo de las industrias exportadoras, el ejemplo más exitoso es la actividad maquiladora de exportación. Estas empresas, entre 1983-1985, tuvieron resultados muy favorables: "Estas industrias se apoyaron en el realismocambiario y en la seguridad de las leyes mexicanas que reglamentan las actividades maquiladoras. Los ingresos de divisas por servicios de transformación de este tipo de empresas manufactureras pasaron de 814.4 millones de dólares en 1983 a 1,153.3 y 1,281.5 millones de dólares en los siguientes dos años, o sea, tuvieron un crecimiento de $50.0 \%$ en dos años, comparable al registrado entre 1979-1981" (Presidencia de la República, 1985: 31).

Es evidente que las relaciones que se mantienen entre México y el exterior, no se limitan a la relación cambiaria descrita; sin embargo, es posible reconocer su función crucial como catalizadora o inhibidora del intercambio (Balassa, 1983: 210-222) o de la producción, como se ha descrito, en base a una serie de condiciones internas y externas. Esta interrelación desborda, como consecuencia, todo intento mecanicista de generalización; sin embargo, para cl caso específico descrito, destaca una relación de naturaleza dialéctica que pone en evidencia la combinación desigual que se establece y aparece cada vez con mayor frecuencia entre espacios nacionales y espacios de organización económica de las firmas.

\section{Las variables microeconómicas.}

El endurecimiento en las condiciones de concurrencia internacional, aunado a la necesidad constante por ensanchar el mercado y mejorar las condiciones de producción en términos de costos de las empresas, hacen patente un perfil de condiciones microeconómicas que se define de la manera siguiente: una marcada tendencia de los inversionistas por reducir los tiempos de recuperación de sus inversiones, de aumentar sus niveles de beneficio y de diversificar sectorial y geográficamente las mismas. Si podemos resumir, esto significa que, por un lado, abiertamente se busca reducir el tiempo de rotación o reciclaje del capital, mientras que por el otro, 
se plantea un desplicgue espacial a través de procesos integradores de localizaciones diferenciadas, que en su conjunto abaten costos y favorecen la posición competitiva de las empresas.

Los intrumentos para materializar tales objetivos son de naturaleza diferente y están estrechamente relacionados; por una parte, son técnicos y orientados a profundizar la división técnica de la producción, que reduzca tiempos c integrc espacios; por la otra, instrumentos de naturaleza organizacional, estrechamente ligados a la inversión y cuyas modalidades han experimentado cambios importantes para responder a las necesidades impuestas por $\mathrm{cl}$ capitalismo actual (Oman, 1984).

Las principales modalidades de inversión internacional en forma no exhaustiva son: riesgo compartido, uso de licencias, franquicias, contratos de gestión, plantas completas, productos en mano, contratos de producción compartida, contratos de riesgo y subcontratación internacional. $^{6}$ Su diversidad explica las condiciones espaciotemporales requeridas para la expansión del capitalismo mundial; sin embargo, es necesario destacar la concentración de las inversiones de riesgo compartido (joint ventures) y la subcontratacion internacional (international subcontracting). Estas modalidades pueden explicar de alguna manera, la naturaleza de las estrategias nacionales de desarrollo y su impacto regional, por medio de la sugerente diferencia que L. Sklair ${ }^{7}$ establece entre "zonas económicas" y "zonas de desarrollo", y que derivan del proceso global de transnacionalización descrito (Sklair, 1988).

En México, hasta antes de 1984, existía una duplicidad en los reglamentos relativos a la inversión extranjera: por un lado, un tratamiento general que limitaba la participación minoritaria de los extranjeros en las empresas nacionales, estableciendo límites en base

${ }^{6}$ Respectivamente: joint ventures, licensing agreements, franchising management contracts, turnkey contracts, product in-hand, production-sharing contract, risk service contracts, international subcontracting.

${ }^{7}$ Es importante establecer una diferencia entre ambas a partir de la estrategia de inversión utilizada. Una zona económica nueva es tođa aquella región olugar en đonde se desarrollan actividades, preferentemente industriales, pero cuya actividad no crea encadenamientos importantes (hacia atrás y hacia adelante). Una zona de desarrollo es aquella que, bajo la definición tradicional, crea nuevas actividades en base a los encadenamientos producidos; en términos de formas de inversión, la diferencia aparece respectivamente entre lo que es una zona cuya actividad primordialmente se basa en la "subcontratación" y aquella en la que tienden a desarrollarse inversiones de "riesgo compartido". 
al criterio del interés nacional, y cuya participación, del 4 al $5 \%$ del total de la inversión fija, se localizaba en sectores claves de la industria, como computadoras, automóviles y laboratorios farmacéuticos; por otro lado, desde 1965 ha existido un tratamiento excepcional para la industria maquiladora, empresas que ensamblan oproducen partes, ya que pueden tener el $100 \%$ de la participación de extranjeros, que en su mayoría son de los Estados Unidos. Legalmente, desde su origen este proceso se apoya en la legislación mexicana especial para este tipo de empresas y que se implementa por la legislación aduanera estadounidense que afecta únicamente el valor agregado de los productos reimportados. Técnica y económicamente, estas industrias se desarrollan por el beneficio que representa una mano de obra barata y calificada, por la localización geográfica que, a pesar de su generalización a todo el territorio nacional, han continuado creciendo en la frontera y por la reorientación en la política comercial de la administración de Miguel de la Madrid, acompañada de cambios en la aplicación de la política de inversión extranjera después de 1982.

El proceso dual descrito, en el caso de México, patentiza la complejidad que en fechas recientes adquiere el diseño y manejo de la política regional en la mayoría de los países, toda vez que la modernización en los procesos industriales, su despliegue y la cuasiinstantaneidad con la que se producen, hacen más vulnerable cualquier acción de naturaleza estructural-regional que se aplique; lo que sugiere la necesidad de una planeación y gestion regional sustentada en la combinación de políticas estructurales y coyunturales, que limiten los desequilibrios locales que acarrea consigo la reorganización de la producción a nivel mundial.

\section{EFECTOS}

$\mathrm{El}$ análisis del impacto regional como resultado del despliegue de la producción mundial, en el caso específico de México se apoya en dos elementos: el primero, trata de identificar el relevo que se desarrolla entre la industria manufacturera y la maquiladora a nivel nacional, con el objeto de darle sentido a dicho proceso; el segundo, que ubica las transformaciones en la composición de ramas en la industria maquiladora, que de manera preliminar permitan identificar las tendencias del comportamiento regional. 
El relevo industrial.

En los últimos años se observa una clara tendencia a generalizar, en el resto del territorio nacional, el patrón de industrialización adjudicado tradicionalmente a la frontera. Este proceso se desarrolla en la medida en que gradualmente se va agotando el modelo de sustitución de importaciones, que concentraba el grueso de su actividad en los principales centros metropolitanos del país.

Este relevo de la industria nacional, efectuado por la maquiladora y las nuevas actividades cimentadas en inversiones extranjeras para la exportación, integra la industria mexicana a la producción mundial, y puede ser aproximado a través de los índices de empleo y de horashombre trabajadas en la industria manufacturera nacional en relación a la industria maquiladora en el período que va de 1975-1986.

La figura 2 destaca, en primer lugar, la relación inversa que se observa en las curvas de tendencia de la industria manufacturera y la maquiladora, tanto en empleo como en horas-hombre trabajadas; en segundo lugar, derivado de este comportamiento, puede distinguirse el reacomodo entre ambos grupos de industrias en el período que va de 1978-1982, cuyas características son una sobrevaluación del peso, un crecimiento muy alto de la producción nacional por la expansión experimentada por la demanda interna en dicho período y una recesión de la economía norteamericana que llega hasta 1981. En conclusión, puede señalarse cómo en ambos subperíodos se destaca la importancia que juega indirectamente la paridad cambiaria en la expansión o contracción de ambos grupos de industrias.

La gráfica 3 muestra con mayor desagregación, el comportamiento de la industria maquiladora en los municipios fronterizos y nofronterizos, de la que se puede hacer dos observaciones: la primera, una vez más el cambio en la tendencia que había venido experimentando la manufactura nacional; la segunda, el crecimiento relativamente mayor que experimenta la industria maquiladora no-fronteriza después de 1982, que confirmaría parcialmente nuestra hipótesis de una "fronterización" del país.

Una conclusión preliminar acerca de la tendencia observada por el crecimiento proporcionalmente mayor de la maquiladora nofronteriza en este último período -cuyo volumen absoluto es aún muy bajo-, es destacar lo que significaría en un futuro próximo la generalización del proceso, siempre que se mantengan cuando menos las siguientes condiciones básicas: transparencia en la política de 


\section{LA RECIENTE INSERCION INTERNACIONAL DE LA INDUSTRIA}
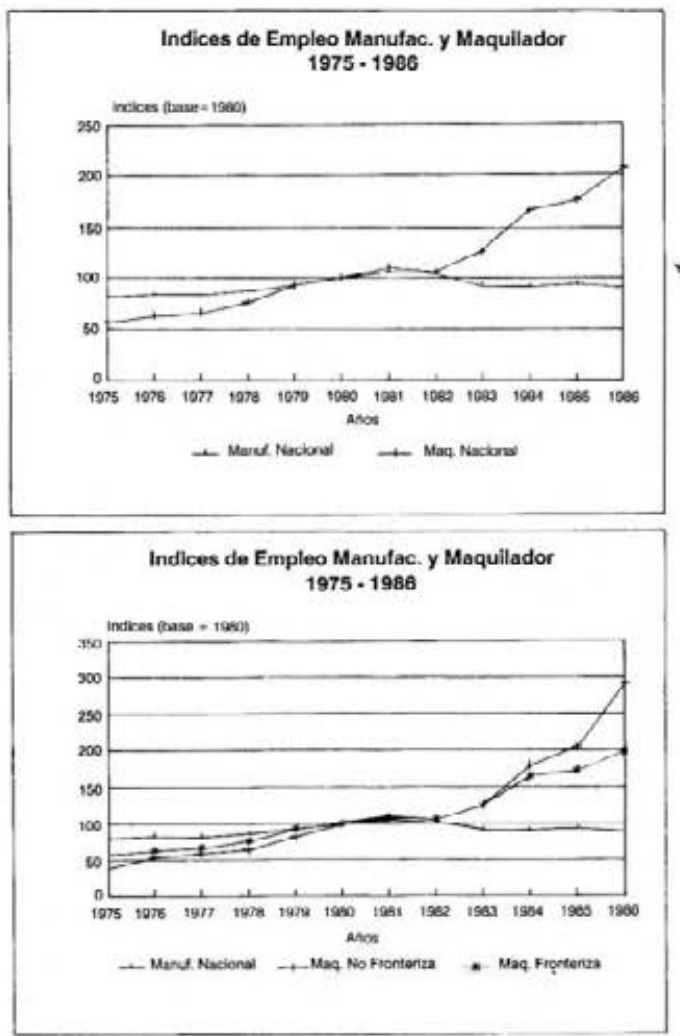

FUENTE: INEGI, Est Ind Maq Exp 75-86

FIGURA 2. Indices de empleo manufacturero y maquilador (1975-1986)

inversiones extranjeras, paridad subvaluada y crecimiento sostenido del consumo en Estados Unidos.

La reorganización espacio-sectorial de la maquiladora.

Una vez relativamente confirmado el despliegue horizontal de las nuevas modalidades de inversión y producción mundial en México, es convenienteverificar el despliegue vertical que se desarrolla en estas actividades para las que contamos con un período más reducido, que va de 1979-1986. Regionalmente, la dimensión espacio-temporal es la más afectada, por ser un producto de la combinación del 
desplazamiento horizontal (geográfico) y la organización sectorial (entre ramas). Según los resultados derivados de los cuadros 4 y 5 , en donde se presentan los índices de empleo de municipios fronterizos y no-fronterizos, se pueden hacer las siguientes observaciones: en primer lugar, se infiere un desplazamiento de actividades ligadas al vestido y textiles hacia el interior del país, asimismo, se desarrolló un crecimiento muy importante en equipo y material de transporte; en segundo lugar, se observa una importante industria de equipo de transporte que continúa su desarrollo en la frontera, y la presencia de un significativo volumen de empleo de materiales y accesorios eléctricos.

CUADRO 4. Indices de empleo por rama de actividad maquiladora 1979-1986 Municipios fronterizos

\begin{tabular}{|c|c|c|c|c|c|c|c|c|}
\hline Rama & 1979 & 1980 & 1981 & 1982 & 1983 & 1984 & 1985 & 1986 \\
\hline Prods. alim. & 106.32 & 100.00 & 112.85 & 116.15 & 136.25 & 125.84 & 133.17 & 156.86 \\
\hline Vestido y textiles & 104.46 & 100.00 & 100.15 & 83.41 & 90.38 & 106.35 & 105.84 & 118.43 \\
\hline Calz. y Cuero & 100.98 & 100.00 & 118.94 & 133.44 & 181.52 & 238.28 & 282.69 & 283.41 \\
\hline Madera y metal & 108.82 & 100.00 & 102.31 & 95.86 & 150.24 & 196.05 & 206.20 & 204.52 \\
\hline P. químicos & n.d. & 100.00 & 96.39 & n.d. & n.d. & n.d. & n.d. & n.d. \\
\hline Eq. $\operatorname{transp}$. & 70.92 & 100.00 & 142.37 & 16249 & 264.99 & 394.93 & 520.82 & 606.41 \\
\hline $\begin{array}{l}\text { Herr. y eq. } \\
\text { expto. eléctr. }\end{array}$ & 100.00 & 100.00 & 76.44 & 72.36 & 82.55 & 117.45 & 130.10 & 177.37 \\
\hline $\begin{array}{l}\text { Maq. e. ap. y art. } \\
\text { clec. y electr. }\end{array}$ & 96.65 & 100.00 & 111.27 & 107.72 & 116.36 & 145.87 & 136.44 & 149.11 \\
\hline $\begin{array}{l}\text { Mat. y acces. } \\
\text { elec. y electr. }\end{array}$ & 91.60 & 100.00 & 110.16 & 106.30 & 119.30 & 159.01 & 145.97 & 160.69 \\
\hline $\begin{array}{l}\text { Art. deport. } \\
\text { y juguetes }\end{array}$ & 87.55 & 100.00 & 95.11 & 91.51 & 124.05 & 220.19 & 259.19 & 253.66 \\
\hline Otras ind. manuf. & 91.45 & 100.00 & 99.57 & 92.85 & 99.57 & 129.03 & 166.68 & 217.71 \\
\hline Servicios & 97.49 & 100.00 & 105.91 & 121.12 & 167.80 & 189.17 & 231.68 & 270.71 \\
\hline
\end{tabular}

Otra conclusión preliminar nos llevaría a hacer los siguientes señalamientos: primero, se infiere que hay un desplazamiento de las inversiones en la industria textil y del vestido hacia el interior del país, probablemente debido a la condición de un transporte eficiente y barato ${ }^{8}$, segundo, se desarrolla y mantiene una fuerte presencia de una importante actividad vinculada a la producción y ensamble de

${ }^{8}$ No debe dejarse pasar por alto el relevante crecimiento experimentado por la industria maquiladora textil en Aguascalientes durante los últimos años; entre sus ventajas está el ser centro geográfico del sistema ferroviario. 
materiales y accesorios elèctricos en la frontera, que probablemente responde a la procesos técnicos geográficamente contiguos; tercero, tanto en la frontera como en el interior, existe un despliegue de material y equipo de transporte, que posiblemente se oriente a convertir al país en un verdadero centro de la producción automotriz mundial.

CUADRO 5. Indices de empleo por rama de actividad maquiladora 1979-1986 Municipios no fronterizos

\begin{tabular}{|c|c|c|c|c|c|c|c|c|}
\hline Rama & 1979 & 1980 & 1981 & 1982 & 1983 & 1984 & 1985 & 1986 \\
\hline $\begin{array}{l}\text { Prods. alim. } \\
\text { Vestido y textiles }\end{array}$ & $\begin{array}{l}\text { n.d. } \\
82.65\end{array}$ & $\begin{array}{l}\text { n.d. } \\
100.00\end{array}$ & $\begin{array}{l}\text { n.d. } \\
114.09\end{array}$ & $\begin{array}{l}\text { n.d. } \\
93.87\end{array}$ & $\begin{array}{l}\text { n.d. } \\
100.39\end{array}$ & $\begin{array}{l}\text { n.d. } \\
142.64\end{array}$ & $\begin{array}{l}\text { n.d. } \\
192.64\end{array}$ & $\begin{array}{c}\text { n.d. } \\
254.32\end{array}$ \\
\hline Calz. y cuero & 42.58 & 100.00 & n.d. & n.d. & n.d. & 111.33 & 79.30 & 82.81 \\
\hline $\begin{array}{l}\text { Madera y metal } \\
\text { P. químicos }\end{array}$ & $\begin{array}{c}108.96 \\
\text { n.d. }\end{array}$ & $\begin{array}{l}100.00 \\
\text { n.d. }\end{array}$ & $\begin{array}{c}123.88 \\
\text { n.d. }\end{array}$ & $\begin{array}{l}67.16 \\
\text { n.d. }\end{array}$ & $\begin{array}{l}202.99 \\
\text { n.d. }\end{array}$ & n.d. & $\begin{array}{l}\text { n.d. } \\
\text { n.d. }\end{array}$ & $\begin{array}{l}\text { n.d. } \\
\text { n.d. }\end{array}$ \\
\hline Eq. transp. & n.d. & 100.00 & 222.75 & 187.75 & 195.00 & 334.50 & 791.75 & 1498.25 \\
\hline $\begin{array}{l}\text { Herr. y eq. } \\
\text { expto. eléctr. }\end{array}$ & n.d. & n.d. & n.d. & n.d. & n.d. & n.d. & n.d. & n.d. \\
\hline $\begin{array}{l}\text { Maq. c ap. y art. } \\
\text { elec. y electr. }\end{array}$ & 89.28 & 100.00 & 133.58 & 196.82 & 294.39 & 492.55 & 400.50 & SS3.85 \\
\hline $\begin{array}{l}\text { Mat. y acces. } \\
\text { elec. } y \text { electr. }\end{array}$ & 66.98 & 100.00 & 96.05 & 87.55 & 97.06 & 125.18 & 133.51 & 163.57 \\
\hline $\begin{array}{l}\text { Art. deport. } \\
\text { y juguetes }\end{array}$ & n.d. & n.d. & n.d. & n.d. & n.d. & n.d. & n.d. & n.d. \\
\hline Otras ind. manu. & 224.58 & 100.00 & 153.01 & 16241 & 229.64 & 288.92 & 344.82 & 738.55 \\
\hline Servicios & 148.74 & 100.00 & 137.08 & 126.49 & 107.82 & 117.69 & 144.17 & 243.11 \\
\hline
\end{tabular}

\section{CONCLUSIONES}

De una manera general, el proceso dual descrito patentiza la complejidad que en fecha reciente adquiere el diseño y manejo de la política regional en la mayoría de los países, toda vez que la modernización en los procesos industriales, su despliegue y la cuasiinstantaneidad con la que éstas se producen, hace más vulnerable cualquier acción de naturaleza estructural que se pudiera aplicar. Esto sugiere la necesidad de una planeación y gestión regional, sustentada en la combinación de políticas estructurales y coyunturales que limiten los desequilibrios locales que lleva consigo la reorganización de la producción a nivel mundial, y que plantea la necesidad de profundizar en los mecanismos en los cuales se realiza la inversión.

De una manera específica, en el plano espacio-sectorial, requiere destacarse el papel de la industria automotriz del país, por dos razones: 
la primera, estrictamente ligada a las profundas transformaciones experimentadas por esta industria a nivel mundial, por los cambios en su organización de producción y distribución a escala mundial, que constituyen el recurso para permanecer en el mercado de las cada vez menos numerosas grandes corporaciones automotrices; la segunda, por las transformaciones en la organización de la producción y de su distribución geográfica en el país, que regionalmente da claras señales de la tendencia a concentrarse "... en una producción masiva con la adaptación de un producto con características mundiales que se concentraría específicamente en las entidades del norte y algunas del centro" (Sánchez L., 1987: 132). Esta tendencia tiene un impacto regional nuevo y diferente a la que posee una maquila tradicional en uso intensivo de trabajo que se desplaza hacia el interior del país, como en el caso de los textiles o una maquila ligada a procesos sofisticados que por su naturaleza no puede alejarse mucho de la frontera (como sucede con la electrónica y high tech). Esta diferencia radica en que la nueva industria automotriz en México está orientada predominantemente al mercado mundial y se localiza, a diferencia de la anterior, entre la frontera y el centro del país, la cual podríamos denominar como centro-norte.

Lo anterior constituye regional y sectorialmente, un dato nuevo que afecta las divisiones precedentes entre las actividades fronterizas y las confinadas al interior del país, por su orientación al mercado interno. Lo que refleja en primer lugar, la complejidad que adquiere la inserción de la industria mexicana en la economía mundial en los últimos años; y en segundo, lapresencia de una actividad industrial orientada hacia la exportación, con fuertes inversiones en volumen y horizonte temporal que tienen un impacto regional significativo en los estados de la franja centro-norte -en donde se localizan las plantas de la industria terminal- y en algunas localidades fronterizas y del centro del país, en donde se ubica la producción de partes y accesorios en un númcro importante de plantas y talleres. 


\section{B I B L I O G R A F I A}

BALASSA, B. 1983."La política de comercio exterior de México". En: Comercio exterior. vol. 33 No. 3.

BASILE, A. Y GERMIADIS D. 1984. Investing in free export processing zones. OCDE. París.

CARNEY, J. Et al. 1980. Regions in crisis. Ed. Crom Helm. Londres

COATSWORTH, J.H. 1984. El impacto económico de los ferrocarriles en el porfiriato. Ed. Era, México.

FROBEL, F.Et al. 1981 La nueva división internacional del trabajo. Siglo XXI Eds. México.

GERMIADIS, D. y MICHALET, Ch.-A. 1984. Intemational banks and financial markets in developing countries. OCDE, París.

GUILLEN ROMO, H. 1988. Origenes de la crisis en México 1942-1982 Ed. Era. México.

HUDSON, R. y SADLER,E. 1986."Contesting work closures in Western Europe's old industrial regions: defending place or betrayin class. En: SCOTT, A.J.y STORPER, M. Production, work, territory. Ed. Allen and Unwin. Londres.

INEGI. 1986. Sistema de cuentas nacionales. México.

KAPP, B. 1974. Les relations exèrieures du Mexique (1821-1921) d'aprés les sources francaises. Ed. Keinecsieck. París.

OECD. 1988a. Structural adjustment and economic performance. OCDE. París.

.--- 1988b. The newly industrialising countries.. OCDE. París.

OMAN, Ch. 1984. New forms in international investment in developing countries. OCDE. París.

PRESIDENCIA DE LA REPUBLICA. 1985 Las razones y las obras, crónica del sexenio 1982-1988. F.C.E. México.

SANCHEZ L., R. 1987. "Hacia una identificación regional de la industria automotriz para los años de 1970-75 y 1980". Tesis profesional. Facultad de Economía. UNAM. México.

SANDEZ P., A. y AVILES M.,Ana Ma. 1988 "Tendencias del crecimiento Industrial fronterizo: el sistema de ciudades de Baja California". Ponencia presentada en el Pacific Coast Council on Latin American Studies. Mexicali, B.C. octubre 20-23. mimeo.

SKLAIR,L. 1988." ¿Representa el programa de las maquiladoras mexicanas una verdadera estrategia de desarrollo? Ponencia presentada en el seminario de investigación sobre las 
relaciones México-Estados Unidos. Centro de Estudios Mexicoamericanos. UCSD. (Aparece en este número de Estudios Fronterizos).

STEINDL, J. 1986. "Problemas estructurales en la crisis actual". En: Investigación económica” No. 176.

TAYLOR, G. R. 1951. The transportation revolution. Rinehart, New York.

TREJO, Saúl. 1987. El futuro de la política industrial en México. El Colegio de México. México. 